\title{
Stochastic modelling of intermittent fluctuations in the scrape-off layer: Correlations, distributions, level crossings, and moment estimation
}

\author{
O. E. Garcia, ${ }^{1, a)}$ R. Kube, ${ }^{1}$ A. Theodorsen, ${ }^{1}$ and H. L. Pécseli ${ }^{2}$ \\ ${ }^{1}$ Department of Physics and Technology, UiT The Arctic University of Norway, N-9037 Troms $\phi$, Norway \\ ${ }^{2}$ Physics Department, University of Oslo, PO Box 1048 Blindern, N-0316 Oslo, Norway
}

(Received 18 February 2016; accepted 6 May 2016; published online 24 May 2016)

\begin{abstract}
A stochastic model is presented for intermittent fluctuations in the scrape-off layer of magnetically confined plasmas. The fluctuations in the plasma density are modeled by a super-position of uncorrelated pulses with fixed shape and duration, describing radial motion of blob-like structures. In the case of an exponential pulse shape and exponentially distributed pulse amplitudes, predictions are given for the lowest order moments, probability density function, auto-correlation function, level crossings, and average times for periods spent above and below a given threshold level. Also, the mean squared errors on estimators of sample mean and variance for realizations of the process by finite time series are obtained. These results are discussed in the context of single-point measurements of fluctuations in the scrape-off layer, broad density profiles, and implications for plasma-wall interactions due to the transient transport events in fusion grade plasmas. The results may also have wide applications for modelling fluctuations in other magnetized plasmas such as basic laboratory experiments and ionospheric irregularities. Published by AIP Publishing.

[http://dx.doi.org/10.1063/1.4951016]
\end{abstract}

\section{INTRODUCTION}

Cross-field transport of particles and heat in the scrapeoff layer (SOL) of non-uniformly magnetized plasmas are often found to be dominated by the radial motion of fieldaligned filaments with excess particles and heat compared to the ambient plasma. ${ }^{1-5}$ These filaments appear as blobs of plasma in the plane perpendicular to the magnetic field and may propagate across the SOL to the main chamber walls. ${ }^{6-10}$ The average SOL plasma density and the particle and heat fluxes due to the radial motion of plasma filaments depend on their velocity, amplitude, and frequency of occurrence. ${ }^{11-15}$ The turbulence-driven transport results in broad plasma profiles in the SOL and enhanced levels of plasma-wall interactions that may be an issue for the next generation plasma confinement experiments and future fusion power reactors. ${ }^{16-33}$

The radial motion of blob-like structures leads to largeamplitude bursts in single-point measurements. The statistical properties of these bursts in the SOL of several tokamak plasmas have recently been elucidated by the analysis of long data time series from probe measurements and gas puff imaging. ${ }^{11-15}$ Conditional averaging has revealed exponentially distributed burst amplitudes and waiting times, while the burst duration is found to be constant. The wave-form for largeamplitude bursts in the time series is well described by an exponential function. ${ }^{11-15,23-32}$ The present study incorporates these features in a stochastic model for intermittent plasma fluctuations in the SOL, described as a super-position of uncorrelated pulses. ${ }^{34-39}$ This model explains many of the salient experimental findings and empirical scaling relations, including broad plasma profiles and large fluctuation levels,

a)odd.erik.garcia@uit.no skewed and flattened probability density functions, and a parabolic relation between the skewness and flatness moments. The latter has been observed in the boundary region of numerous experiments on magnetized plasmas. ${ }^{11-14,40-44}$

Intermittent fluctuations in the far periphery of magnetically confined plasmas can significantly amplify plasmawall interactions. In order to analyze the intermittent features of the process, level crossing rates and excess time statistics are introduced, that is, a study of the frequency of level crossings and the duration of time intervals where a realization of the process exceeds some prescribed threshold level. ${ }^{45-49}$ This definition will be particularly useful for studies of confinement of hot plasmas, where it may be important to distinguish many short plasma bursts from a few long ones. Although the accumulated time in the bursts can be the same, their consequences will be different as far as, for instance, the heat load on a confining wall is concerned. ${ }^{49}$ The stochastic model is here used to derive expressions for the average time spent above and below a prescribed reference level, and the dependence on the pulse duration and average waiting time is discussed. The analysis makes use of some general results for synthetic time series, often called the Rice model, ${ }^{35}$ which has been used also for modeling plasma fluctuations. ${ }^{36}$ For completeness, the relevant elements of that analysis are summarized here.

This manuscript is organized as follows. In Sec. II, the stochastic model based on a super-position of uncorrelated pulses is presented and its basic predictions are derived for general pulse shapes and amplitude distributions. The cumulants and lower order moments are derived in Sec. III and shown to predict a parabolic relation between the skewness and flatness moments. In the case of an exponential pulse function and exponentially distributed pulse amplitudes, the probability density function is shown in Sec. IV to be Gamma 
distribution with the scale parameter given by the average pulse amplitude and the shape parameter given by the ratio of the pulse duration and average waiting time. In Sec. V, the level crossing rate and average times spent by the process above and below a given threshold level are considered. The mean squared errors on estimates of the mean and variance for realizations of the stochastic process are derived in Sec. VI. Finally, a discussion of the results is presented in Sec. VII and conclusions are given in Sec. VIII.

\section{STOCHASTIC MODEL}

This section begins with a motivation of the stochastic model based on the well-known properties of blob motion in the SOL. This is followed by a discussion of some basic properties and predictions of the stochastic model, including the mean, variance, auto-correlation function, and power spectral density.

\section{A. Super-position of pulses}

Consider the case of plasma fluctuations due to a superposition of $K$ pulses which propagate in space without change of shape, ${ }^{36}$

$$
\Phi_{K}(x, t)=\sum_{k=1}^{K} A_{k} \varphi_{k}\left(x-x_{k}-v_{k} t\right),
$$

where for the $k$-th pulse $A_{k}$ is the pulse amplitude and $\varphi_{k}(x)$ denotes the pulse shape which propagate with velocity $v_{k}$ along the $x$-axis. At time $t=0$, the pulse labeled $k$ is located at $x_{k}$. A single-point measurement at some given position $\xi$ will record the pulse events at various times, giving the signal

$$
\Phi_{K}(\xi, t)=\sum_{k=1}^{K} A_{k} \varphi_{k}\left(v_{k}\left(t_{k}-t\right)\right)
$$

where the reference time for pulse $k$ is given by $t_{k}=\left(\xi-x_{k}\right) /$ $v_{k}$. As an example, consider a pulse shape with a steep front and a trailing wake defined by

$$
\varphi_{k}(x)=\Theta\left(-\frac{x}{\ell_{k}}\right) \exp \left(\frac{x}{\ell_{k}}\right)
$$

where $\ell_{k}$ is the pulse size and the unit step function is defined by

$$
\Theta(y)= \begin{cases}0, & y<0 \\ 1, & y \geq 0\end{cases}
$$

The signal recorded at position $\xi$ is in this case given by

$$
\Phi_{K}(\xi, t)=\sum_{k=1}^{K} A_{k} \Theta\left(\frac{v_{k}\left(t-t_{k}\right)}{\ell_{k}}\right) \exp \left(-\frac{v_{k}\left(t-t_{k}\right)}{\ell_{k}}\right) .
$$

The pulse duration for event $k$ is here given by the transit time $\tau_{\mathrm{d}}=\ell_{k} / v_{k}$. In the following, the properties of the time series in Eq. (5) will be investigated assuming a constant pulse duration $\tau_{\mathrm{d}}$. Further discussion on the filament motion is given in Sec. VII.

Based on the foregoing discussion of pulse propagation, it is of interest to consider a stochastic process given by the super-position of a random sequence of $K$ pulses $^{34-39}$

$$
\Phi_{K}(t)=\sum_{k=1}^{K(T)} A_{k} \varphi\left(t-t_{k}\right)
$$

where for event $k, t_{k}$ is the pulse arrival time, $A_{k}$ is the pulse amplitude, and $\varphi(t)$ is some prescribed pulse shape which is taken to be the same of all events. In Eq. (6), the sum is over exactly $K$ pulses present in a record of duration $T$. In the following, it is assumed that $T$ is large compared with the range of values of $t$ for which $\varphi(t)$ is appreciably different from zero, thus allowing to neglect end effects in a given realization of the process.

The pulse duration time, in general defined by

$$
\tau_{\mathrm{d}}=\int_{-\infty}^{\infty} \mathrm{d} t|\varphi(t)|
$$

is assumed to be finite and taken to be the same for all pulses in the process. The definition in Eq. (7) is consistent with the special example of $\tau_{\mathrm{d}}$ discussed before. The integral of the $n$ th power of the pulse shape will appear frequently in the following and is defined as:

$$
I_{n}=\frac{1}{\tau_{\mathrm{d}}} \int_{-\infty}^{\infty} \mathrm{d} t[\varphi(t)]^{n}
$$

This integral is assumed to be finite for all integers $n \geq 1$. Note that when the pulse shape is an odd function, $I_{1}=0$, while if $\varphi(t)$ is non-negative $I_{1}=1$.

The pulse function described by Eq. (5) corresponds to the one-sided exponential function

$$
\varphi(t)=\Theta\left(\frac{t}{\tau_{\mathrm{d}}}\right) \exp \left(-\frac{t}{\tau_{\mathrm{d}}}\right)
$$

This special case is readily generalized to a doubleexponential pulse shape with a finite rise time, but for simplicity only the one-sided exponential pulse will be considered in the following. The integral of the $n$-th power of the pulse shape is then given by

$$
I_{n}=\frac{1}{\tau_{\mathrm{d}}} \int_{0}^{\infty} \mathrm{d} t \exp \left(-\frac{n t}{\tau_{\mathrm{d}}}\right)=\frac{1}{n} .
$$

Although this relation is used repeatedly in the following, it should be noted that many of the results given below are independent of the details of the pulse shape.

The pulse arrival times $t_{k}$ are in the following assumed to be uniformly distributed on a large interval $T$, so that any particular pulse labeled $k$ is just as likely to arrive at one time as it is at any other time, that is, the pulse arrival time probability density function is the uniform distribution $P_{t}\left(t_{k}\right)=1 / T$. Thus, the probability that $t_{k}$ lies in the interval $(t, t+\mathrm{d} t)$ is $\mathrm{d} t / T$, irrespective of the arrival of any other pulse. With these assumptions, the conditional probability 
that there are exactly $K$ pulse arrivals during any interval of duration $T$ is given by the Poisson distribution

$$
P_{K}(K \mid T)=\frac{1}{K !}\left(\frac{T}{\tau_{\mathrm{w}}}\right)^{K} \exp \left(-\frac{T}{\tau_{\mathrm{w}}}\right),
$$

where $\tau_{\mathrm{w}}$ is the average pulse waiting time. The average number of pulses in a record of duration $T$ is

$$
\langle K\rangle=\sum_{K=0}^{\infty} K P_{K}(K \mid T)=\frac{T}{\tau_{\mathrm{w}}},
$$

where here and in the following, angular brackets denote the ensemble average of a random variable over all its arguments. From the Poisson distribution, it follows that the waiting time between two subsequent pulses is exponentially distributed. From these underlying properties of the stochastic model, it is possible to derive analytical predictions in a closed form for the fundamental fluctuation statistics. This is the main objective of the present contribution.

\section{B. Mean and variance}

The mean value of $\Phi_{K}(t)$ is given by averaging over all random variables. Starting with the case of exactly $K$ events in a realization with duration $T$, this gives ${ }^{35}$

$$
\begin{aligned}
\left\langle\Phi_{K}\right\rangle= & \int_{0}^{T} \frac{\mathrm{d} t_{1}}{T} \int_{-\infty}^{\infty} \mathrm{d} A_{1} P_{A}\left(A_{1}\right) \cdots \int_{0}^{T} \frac{\mathrm{d} t_{K}}{T} \\
& \times \int_{-\infty}^{\infty} \mathrm{d} A_{K} P_{A}\left(A_{K}\right) \sum_{k=1}^{K} A_{k} \varphi\left(t-t_{k}\right),
\end{aligned}
$$

using that the pulse arrival times are uniformly distributed. It is assumed that all $K$ pulses arrive in the interval $(0, T)$. This naturally leads to a non-stationary transient for small observation times $t$ where few pulses contribute to the signal. Similarly, for large observation times, $t>T$, no new pulses arrive and the signal $\Phi_{K}(t)$ eventually decays at the same rate as the individual pulses. In order to quantify this nonstationarity, the integral above is calculated by a simple change of variables giving

$$
\begin{aligned}
& \int_{0}^{T} \mathrm{~d} t_{k} \varphi\left(t-t_{k}\right) \\
& \quad=\int_{0}^{T} \mathrm{~d} t_{k} \Theta\left(\frac{t-t_{k}}{\tau_{\mathrm{d}}}\right) \exp \left(-\frac{t-t_{k}}{\tau_{\mathrm{d}}}\right) \\
& \quad=\tau_{\mathrm{d}} \begin{cases}0, & t \leq 0, \\
1-\exp \left(-\frac{t}{\tau_{\mathrm{d}}}\right), & 0<t \leq T, \\
\exp \left(-\frac{t-T}{\tau_{\mathrm{d}}}\right)-\exp \left(-\frac{t}{\tau_{\mathrm{d}}}\right), & t>T,\end{cases}
\end{aligned}
$$

for a pulse with arrival time $t_{k}$. For observation times $t<0$, the mean value of the signal evidently vanishes since all pulses arrive in the interval $(0, T)$. For observation times within the interval $(0, T)$, the mean value transiently increases and the process is stationary only on temporal scales much longer than the pulse duration, $t \gg \tau_{\mathrm{d}}$. This is due to the transient building up of the signal at small times where few pulses contribute to the mean value. For observation times longer than the record under consideration, $t>T$, the signal $\Phi_{K}(t)$ decreases due to the absence of new pulse arrivals and this naturally influences the mean value.

Neglecting end effects due to the finite duration of individual pulses by taking the integration limits in Eq. (13) to infinity, the mean value of the signal follows directly,

$$
\left\langle\Phi_{K}\right\rangle=\langle A\rangle \frac{K}{T} \int_{-\infty}^{\infty} \mathrm{d} t \varphi(t)=\tau_{\mathrm{d}} I_{1}\langle A\rangle \frac{K}{T} .
$$

Assuming that the number of pulses $K$ is given by a Poisson distribution, it follows that the mean value of the stationary process is given by

$$
\langle\Phi\rangle=\sum_{K=0}^{\infty}\left\langle\Phi_{K}\right\rangle P_{K}(K \mid T)=\frac{\tau_{\mathrm{d}}}{\tau_{\mathrm{w}}} I_{1}\langle A\rangle .
$$

For a non-negative pulse function, $I_{1}=1$, the mean value of the process is given by the average pulse amplitude and the ratio of the pulse duration and average waiting time. Note that the mean value vanishes for anti-symmetric pulse shapes, $I_{1}=0$, and for pulse amplitude distributions with zero mean, $\langle A\rangle=0$.

Similarly, the variance can be calculated by using the relation $\Phi_{\mathrm{rms}}^{2}=\left\langle(\Phi-\langle\Phi\rangle)^{2}\right\rangle=\left\langle\Phi^{2}\right\rangle-\langle\Phi\rangle^{2}$, where $\Phi_{\mathrm{rms}}$ denotes the root mean square (rms) value of $\Phi$. From the definition of $\Phi_{K}(t)$, it follows that

$$
\Phi_{K}^{2}(t)=\sum_{k=1}^{K} \sum_{\ell=1}^{K} A_{k} A_{\ell} \varphi\left(t-t_{k}\right) \varphi\left(t-t_{\ell}\right) .
$$

Averaging this over pulse amplitudes and arrival times for fixed $t$ and $K$ as above gives

$$
\begin{aligned}
\left\langle\Phi_{K}^{2}\right\rangle= & \int_{0}^{T} \frac{\mathrm{d} t_{1}}{T} \int_{-\infty}^{\infty} \mathrm{d} A_{1} P_{A}\left(A_{1}\right) \cdots \int_{0}^{T} \frac{\mathrm{d} t_{K}}{T} \int_{-\infty}^{\infty} \mathrm{d} A_{K} P_{A}\left(A_{K}\right) \\
& \times \sum_{k=1}^{K} \sum_{\ell=1}^{K} A_{k} A_{\ell} \varphi\left(t-t_{k}\right) \varphi\left(t-t_{\ell}\right) .
\end{aligned}
$$

There are two contributions to the variance from the double sum. When $k=\ell$, there are $K$ terms given by the integral

$$
\int_{0}^{T} \frac{\mathrm{d} t_{k}}{T} \int_{-\infty}^{\infty} \mathrm{d} A_{k} P_{A}\left(A_{k}\right) A_{k}^{2} \varphi^{2}\left(t-t_{k}\right),
$$

while for $k \neq \ell$ there are $K(K-1)$ terms with the value

$$
\begin{aligned}
& \int_{0}^{T} \frac{\mathrm{d} t_{k}}{T} \int_{-\infty}^{\infty} \mathrm{d} A_{k} P_{A}\left(A_{k}\right) A_{k} \varphi\left(t-t_{k}\right) \\
& \quad \times \int_{0}^{T} \frac{\mathrm{d} t_{\ell}}{T} \int_{-\infty}^{\infty} \mathrm{d} A_{\ell} P_{A}\left(A_{\ell}\right) A_{\ell} \varphi\left(t-t_{\ell}\right) .
\end{aligned}
$$

Again neglecting the end effects due to the finite duration of individual pulses by extending the integration over $t_{k}$ and $t_{\ell}$ to infinity, the variance for large $T$ is given by 


$$
\begin{aligned}
\left\langle\Phi_{K}^{2}\right\rangle= & \frac{K}{T}\left\langle A^{2}\right\rangle \int_{-\infty}^{\infty} \mathrm{d} t[\varphi(t)]^{2} \\
& +\frac{K(K-1)}{T^{2}}\langle A\rangle^{2}\left[\int_{-\infty}^{\infty} \mathrm{d} t \varphi(t)\right]^{2} .
\end{aligned}
$$

By averaging over all realizations where the number of pulses $K$ is statistically distributed, using that $\langle K(K-1)\rangle=\langle K\rangle^{2}$ for a Poisson distribution gives

$$
\left\langle\Phi^{2}\right\rangle=\sum_{K=0}^{\infty}\left\langle\Phi_{K}^{2}\right\rangle P_{K}(K \mid T)=\frac{\tau_{\mathrm{d}}}{\tau_{\mathrm{w}}} I_{2}\left\langle A^{2}\right\rangle+\langle\Phi\rangle^{2} .
$$

It follows that the variance is given by

$$
\Phi_{\mathrm{rms}}^{2}=\frac{\tau_{\mathrm{d}}}{\tau_{\mathrm{w}}} I_{2}\left\langle A^{2}\right\rangle
$$

and thus the coefficient of variation, or relative fluctuation level for the process, for a non-zero mean is

$$
\frac{\Phi_{\mathrm{rms}}}{\langle\Phi\rangle}=\left(\frac{\tau_{\mathrm{w}}}{\tau_{\mathrm{d}}}\right)^{1 / 2} \frac{I_{2}^{1 / 2}}{I_{1}} \frac{\left\langle A^{2}\right\rangle^{1 / 2}}{\langle A\rangle} .
$$

The relative fluctuation level increases with increasing waiting time and decreasing pulse duration. The main properties of the stochastic process are thus given by the ratio of the pulse duration and average waiting time,

$$
\gamma=\frac{\tau_{\mathrm{d}}}{\tau_{\mathrm{w}}}
$$

For reasons to become clear, this will be referred to as the intermittency parameter of the model.

The interpretation of the above results is evident. For short waiting times and long pulse durations, $\gamma \gg 1$, there is a significant pulse overlap and the signal will at any time be influenced by many individual pulses. This results in a large mean value and small relative variation. In the opposite limit of long waiting times and short pulse durations, $\gamma \ll 1$, the signal is dominated by the isolated pulse events, resulting in a small mean value and large relative fluctuations. These features of the signal are illustrated in Fig. 1, which shows realizations of

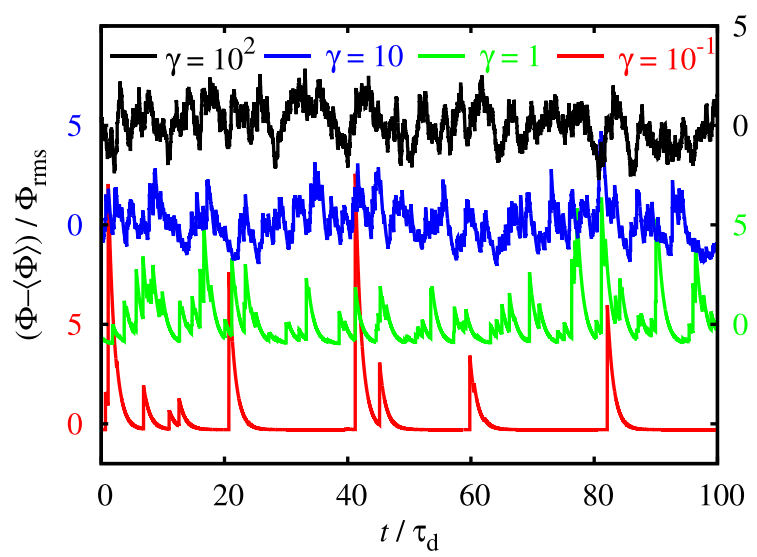

FIG. 1. Realizations of the stochastic process for exponentially distributed pulse amplitudes and waiting times and an exponential pulse shape. The degree of pulse overlap is determined by the intermittency parameter $\gamma=\tau_{\mathrm{d}} / \tau_{\mathrm{w}}$. the process for different values of $\gamma$ based on the exponentially distributed pulse amplitudes and waiting times and an exponential pulse shape as given by Eq. (9). Here, it is clear that for small values of $\gamma$, the signal is dominated by isolated pulses resulting in large-amplitude bursts and strongly skewed fluctuations. For large values of $\gamma$, there is significant overlap of pulse structures and the fluctuations appear symmetric with small relative amplitudes. This observation will be quantified by the calculation of higher order moments and the probability density function of the signal in Secs. III and IV, respectively.

\section{Correlation function}

Considering first the signal $\Phi_{K}(t)$ defined by Eq. (6), the two-point correlation is given by

$$
\begin{aligned}
& \left\langle\Phi_{K}(t) \Phi_{K}(t+\tau)\right\rangle \\
& =\int_{0}^{T} \frac{\mathrm{d} t_{1}}{T} \int_{0}^{\infty} \mathrm{d} A_{1} P_{A}\left(A_{1}\right) \cdots \int_{0}^{T} \frac{\mathrm{d} t_{K}}{T} \\
& \quad \times \int_{0}^{\infty} \mathrm{d} A_{K} P_{A}\left(A_{K}\right) \sum_{k=1}^{K} \sum_{\ell=1}^{K} A_{k} \varphi\left(t-t_{k}\right) A_{\ell} \varphi\left(t-t_{\ell}+\tau\right) \\
& =\left\langle A^{2}\right\rangle \sum_{k=1}^{K} \int_{0}^{T} \frac{\mathrm{d} t_{1}}{T} \varphi\left(t-t_{k}\right) \varphi\left(t-t_{k}+\tau\right) \\
& \quad+\langle A\rangle^{2} \sum_{\substack{k, \ell=1 \\
k \neq \ell}}^{K} \int_{0}^{T} \frac{\mathrm{d} t_{k}}{T} \varphi\left(t-t_{k}\right) \int_{0}^{T} \frac{\mathrm{d} t_{\ell}}{T} \varphi\left(t-t_{\ell}+\tau\right) .
\end{aligned}
$$

Here again, the double sum has been divided into two parts. The first part consists of $K$ terms where $k=\ell$ and the second part consists of $K(K-1)$ terms where $k \neq \ell$. Neglecting the end effects due to the finite pulse duration by taking the integration limits to infinity, the convolution for the one-sided exponential pulse function is

$$
\frac{1}{T} \int_{-\infty}^{\infty} \mathrm{d} t_{k} \varphi\left(t-t_{k}\right) \varphi\left(t-t_{k}+\tau\right)=\frac{\tau_{\mathrm{d}}}{2 T} \exp \left(-\frac{|\tau|}{\tau_{\mathrm{d}}}\right) .
$$

Substituting this result into Eq. (26) and averaging over the number of pulses occurring in the interval of length $T$, it follows that the auto-correlation function for the stationary process $\Phi(t)$ is given by

$$
\begin{aligned}
R_{\Phi}(\tau) & =\langle\Phi(t) \Phi(t+\tau)\rangle \\
& =\sum_{K=0}^{\infty}\left\langle\Phi_{K}(t) \Phi_{K}(t+\tau)\right\rangle P_{K}(K \mid T) \\
& =\langle\Phi\rangle^{2}+\Phi_{\mathrm{rms}}^{2} \exp \left(-\frac{|\tau|}{\tau_{\mathrm{d}}}\right)
\end{aligned}
$$

which is independent of the amplitude distribution $P_{A}$ as far as the mean and variance are finite. The correlation function decreases exponentially with separation $\tau$ with a rate given by the duration of individual pulses.

The power spectral density $S_{\Phi}(\omega)$ is given by the Fourier transform of the auto-correlation function,

$$
S_{\Phi}(\omega)=2 \pi\langle\Phi\rangle^{2} \delta(\omega)+\Phi_{\mathrm{rms}}^{2} \frac{2 \tau_{\mathrm{d}}}{1+\tau_{\mathrm{d}}^{2} \omega^{2}},
$$


where $\omega$ is the angular frequency. The first term in the above equation is the contribution from the mean value of the signal, giving a zero frequency contribution. The second term is a Lorentzian spectrum, independent of frequency for $0<\tau_{\mathrm{d}}^{2} \omega^{2} \ll 1$, and has the asymptotic behavior $1 / \tau_{\mathrm{d}}{ }^{2} \omega^{2}$ for high frequencies $\tau_{\mathrm{d}}^{2} \omega^{2} \gg 1$. It is of interest to note that the average waiting time does not appear explicitly in the functional shape of the auto-correlation function and the power spectral density, which is due to the assumed independence of the pulse events.

\section{CUMULANTS AND MOMENTS}

In this section, a general expression is derived for the characteristic function of the random variable $\Phi$ in the case of a Poisson distribution of pulse events. From this, the cumulants and the lowest order statistical moments are found and shown to predict a parabolic relation between the skewness and flatness moments, the result agreeing with a more general inequality. ${ }^{50}$ Systematic skewness-flatness relations are also found in the studies of, for instance, concentration fluctuations in the neutral atmosphere and seem thus to be of universal nature. ${ }^{51-55}$

\section{A. Characteristic function}

The characteristic function for a sum of independent random variables is the product of their individual characteristic functions. Thus, the conditional probability density $P_{\Phi}(\Phi \mid K)$ that a sum of $K$ pulse events $\phi_{k}$ lies in the range between $\Phi$ and $\Phi+\mathrm{d} \Phi$ is given by

$$
P_{\Phi}(\Phi \mid K)=\frac{1}{2 \pi} \int_{-\infty}^{\infty} \mathrm{d} u \exp (-i \Phi u) \prod_{k=1}^{K}\left\langle\exp \left(i \phi_{k} u\right)\right\rangle
$$

where the characteristic functions $\left\langle\exp \left(i \phi_{k} u\right)\right\rangle$ are averaged over the values of $\phi_{k}=A_{k} \varphi\left(t-t_{k}\right)$. For general amplitude distribution and pulse shape,

$$
\left\langle\exp \left(i \phi_{k} u\right)\right\rangle=\int_{0}^{T} \frac{\mathrm{d} t_{k}}{T} \int_{-\infty}^{\infty} \mathrm{d} A_{k} P_{A}\left(A_{k}\right) \exp \left[i u A_{k} \varphi\left(t-t_{k}\right)\right],
$$

where $T$ is the duration of the time interval under consideration. Since all the $K$ characteristic functions in Eq. (32) are the same, the conditional probability density is

$$
P_{\Phi}(\Phi \mid K)=\frac{1}{2 \pi} \int_{-\infty}^{\infty} \mathrm{d} u \exp (-i \Phi u)\left\langle\exp \left(i \phi_{k} u\right)\right\rangle^{K}
$$

assuming the number of events $K$ in a time interval $T$ to be given. The probability density function for the random variable $\Phi$ is given by

$$
\begin{aligned}
P_{\Phi}(\Phi) & =\sum_{K=0}^{\infty} P_{\Phi}(\Phi \mid K) P_{K}(K \mid T) \\
& =\frac{1}{2 \pi} \int_{-\infty}^{\infty} \mathrm{d} u \exp \left(-i \Phi u+\frac{T}{\tau_{\mathrm{w}}}\left\langle\exp \left(i \phi_{k} u\right)\right\rangle-\frac{T}{\tau_{\mathrm{w}}}\right),
\end{aligned}
$$

where $P_{K}(K \mid T)$ is the Poisson distribution given in Eq. (11). The stationary probability density function for $\Phi$ is obtained by extending the integration limits for $t_{k}$ to infinity. This leads to the desired result

$$
\begin{aligned}
P_{\Phi}(\Phi)= & \frac{1}{2 \pi} \int_{-\infty}^{\infty} \mathrm{d} u \exp \left(-i \Phi u+\frac{1}{\tau_{\mathrm{w}}} \int_{-\infty}^{\infty} \mathrm{d} A P_{A}(A)\right. \\
& \left.\times \int_{-\infty}^{\infty} \mathrm{d} t[\exp (i u A \varphi(t))-1]\right) .
\end{aligned}
$$

According to this equation, the logarithm of the characteristic function of $P_{\Phi}$ is

$$
\begin{aligned}
& \frac{1}{\tau_{\mathrm{w}}} \int_{-\infty}^{\infty} \mathrm{d} A P_{A}(A) \int_{-\infty}^{\infty} \mathrm{d} t[\exp (i u A \varphi(t))-1] \\
& \quad=\sum_{n=1}^{\infty} \frac{1}{\tau_{\mathrm{w}}} \frac{(i u)^{n}}{n !} \int_{-\infty}^{\infty} \mathrm{d} A A^{n} P_{A}(A) \int_{-\infty}^{\infty} \mathrm{d} t[\varphi(t)]^{n},
\end{aligned}
$$

where the exponential function has been expanded in a power series. The cumulants $\kappa_{n}$ are the coefficients in the expansion of the logarithm of the characteristic function for $P_{\Phi}$

$$
\ln \langle\exp (i \Phi u)\rangle=\ln \int_{-\infty}^{\infty} \mathrm{d} \Phi \exp (i \Phi u) P_{\Phi}(\Phi)=\sum_{n=1}^{\infty} \kappa_{n} \frac{(i u)^{n}}{n !} .
$$

For the stochastic process considered here, the cumulants are thus given by

$$
\kappa_{n}=\frac{1}{\tau_{\mathrm{w}}} \int_{-\infty}^{\infty} \mathrm{d} A A^{n} P_{A}(A) \int_{-\infty}^{\infty} \mathrm{d} t[\varphi(t)]^{n}=\gamma I_{n}\left\langle A^{n}\right\rangle,
$$

where $I_{n}$ is defined in Eq. (8). From the cumulants, the lowest order moments are readily obtained. A formal power series expansion shows that the characteristic function is related to the raw moments of $\Phi$, defined by $\mu_{n}^{\prime}=\left\langle\Phi^{n}\right\rangle$

$$
\langle\exp (i \Phi u)\rangle=1+\sum_{n=1}^{\infty} \frac{\langle i \Phi u\rangle^{n}}{n !}=1+\sum_{n=1}^{\infty} \mu_{n}^{\prime} \frac{(i u)^{n}}{n !} .
$$

Further expanding the logarithmic function in Eq. (36) and using Eq. (38), it follows that the lowest order centred moments $\mu_{n}=\left\langle(\Phi-\langle\Phi\rangle)^{n}\right\rangle$ are related to the cumulants by the relations $\mu_{2}=\kappa_{2}, \mu_{3}=\kappa_{3}$ and $\mu_{4}=\kappa_{4}+3 \kappa_{2}^{2}$. From this, the skewness and flatness moments are readily obtained.

\section{B. Skewness and flatness}

Higher order moments are straightforward to calculate from Eq. (37) for general pulse shapes and amplitude distributions. In particular, the skewness and flatness moments are given, respectively, by

$$
\begin{gathered}
S_{\Phi}=\frac{\left\langle(\Phi-\langle\Phi\rangle)^{3}\right\rangle}{\Phi_{\mathrm{rms}}^{3}}=\frac{1}{\gamma^{1 / 2}} \frac{I_{3}}{I_{2}^{3 / 2}} \frac{\left\langle A^{3}\right\rangle}{\left\langle A^{2}\right\rangle^{3 / 2}}, \\
F_{\Phi}=\frac{\left\langle(\Phi-\langle\Phi\rangle)^{4}\right\rangle}{\Phi_{\mathrm{rms}}^{4}}=3+\frac{1}{\gamma} \frac{I_{4}}{I_{2}^{2}} \frac{\left\langle A^{4}\right\rangle}{\left\langle A^{2}\right\rangle^{2}} .
\end{gathered}
$$


The relative fluctuation level, skewness, and flatness all increase with decreasing $\gamma$. For large $\gamma$, the skewness and excess flatness vanish, consistent with a normal distribution of the fluctuations. For these reasons, $\gamma$ is referred to as the intermittency parameter of the model.

The relations given in Eq. (39) imply that there is a parabolic relation between the skewness and flatness moments ${ }^{37}$

$$
F_{\Phi}=3+\frac{I_{2} I_{4}}{I_{3}^{2}} \frac{\left\langle A^{2}\right\rangle\left\langle A^{4}\right\rangle}{\left\langle A^{3}\right\rangle^{2}} S_{\Phi}^{2}
$$

This relation holds for any pulse shape and amplitude distribution as far as the pulse function integrals given by Eq. (8) and the amplitude moments exist.

For the exponential pulse shape given by Eq. (9), the integral in Eq. (37) is trivially calculated by the use of Eq. (10). The cumulants are thus given by $\kappa_{n}=\gamma\left\langle A^{n}\right\rangle / n$. It follows that the variance, like the mean value, depends on the ratio of the pulse duration and average waiting time,

$$
\Phi_{\mathrm{rms}}^{2}=\frac{\gamma}{2}\left\langle A^{2}\right\rangle .
$$

The square of the relative fluctuation level, or coefficient of variation, is accordingly given by

$$
\frac{\Phi_{\mathrm{rms}}^{2}}{\langle\Phi\rangle^{2}}=\frac{1}{2 \gamma} \frac{\left\langle A^{2}\right\rangle}{\langle A\rangle^{2}}
$$

Similarly, the expressions for the skewness and flatness moments simplify to become

$$
\begin{gathered}
S_{\Phi}=\left(\frac{8}{9 \gamma}\right)^{1 / 2} \frac{\left\langle A^{3}\right\rangle}{\left\langle A^{2}\right\rangle^{3 / 2}}, \\
F_{\Phi}=3+\frac{1}{\gamma} \frac{\left\langle A^{4}\right\rangle}{\left\langle A^{2}\right\rangle^{2}} .
\end{gathered}
$$

This demonstrates that the probability density function for $\Phi$ is positively skewed, $S_{\Phi}>0$, and flattened, $F_{\Phi}>3$, for positive definite pulse amplitudes $A$. Moreover, from the foregoing expressions, it follows that the parabolic relation between skewness and flatness simplifies to

$$
F_{\Phi}=3+\frac{9}{8} \frac{\left\langle A^{2}\right\rangle\left\langle A^{4}\right\rangle}{\left\langle A^{3}\right\rangle^{2}} S_{\Phi}^{2}
$$

The above expressions for the lowest order moments are further simplified in the particular case of exponentially distributed pulse amplitudes

$$
P_{A}=\frac{1}{\langle A\rangle} \exp \left(-\frac{A}{\langle A\rangle}\right) .
$$

The raw amplitude moments are then given by $\left\langle A^{n}\right\rangle=n !\langle A\rangle^{n}$. In this case, the relative fluctuation level can be written as

$$
\frac{\Phi_{\mathrm{rms}}}{\langle\Phi\rangle}=\frac{1}{\gamma^{1 / 2}}
$$

while the skewness and flatness moments for $\Phi$ reduce to

$$
\begin{gathered}
S_{\Phi}=\frac{2}{\gamma^{1 / 2}}, \\
F_{\Phi}=3+\frac{6}{\gamma} .
\end{gathered}
$$

The parabolic relation between these moments becomes simply $F_{\Phi}=3+3 S_{\Phi}^{2} / 2$, notably independent of the model parameters.

\section{AMPLITUDE DISTRIBUTION}

In this section, the probability density function for the random variable $\Phi$ will be derived in the case of an exponential pulse shape and exponentially distributed pulse amplitudes. The limits of weak and strong intermittency are discussed in detail.

\section{A. Normal limit}

The results presented above show that the skewness and flatness moments vanish in the limit of large $\gamma$. It can be demonstrated that the probability density function for $\Phi$ then approaches a normal distribution, independent of the details of the pulse shape and amplitude distribution. The distribution $P_{\Phi}$ can be written in terms of the characteristic function given in Eq. (36)

$$
P_{\Phi}(\Phi)=\frac{1}{2 \pi} \int_{-\infty}^{\infty} \mathrm{d} u \exp \left(-i \Phi u+\sum_{n=1}^{\infty} \frac{(i u)^{n} \kappa_{n}}{n !}\right)
$$

where the cumulants are given by Eq. (37). In the limit of large $\gamma$, the exponential function can be expanded as a power series in $u$. Integrating term by term then gives ${ }^{35}$

$$
\begin{aligned}
\lim _{\gamma \rightarrow \infty} \Phi_{\mathrm{rms}} P_{\Phi}(\tilde{\Phi}) \\
=\lim _{\gamma \rightarrow \infty} \frac{1}{(2 \pi)^{1 / 2}} \exp \left(-\frac{\tilde{\Phi}^{2}}{2}\right)\left[1+\frac{\mu_{3}}{3 ! \Phi_{\mathrm{rms}}^{3}(2 \pi)^{1 / 2}}\right. \\
\left.\quad \times\left(\tilde{\Phi}^{3}-3 \tilde{\Phi}\right)+\mathcal{O}(1 / \gamma)\right]
\end{aligned}
$$

where the last term inside the square brackets represent the sum of the remaining terms in the expansion and the centred and scaled amplitude is defined by

$$
\tilde{\Phi}=\frac{\Phi-\langle\Phi\rangle}{\Phi_{\mathrm{rms}}},
$$

with the mean value $\langle\Phi\rangle$ and fluctuation level $\Phi_{\text {rms }}$ given by Eqs. (16) and (23), respectively. The terms inside the square brackets in Eq. (49) are of order $1,1 / \gamma^{1 / 2}$, and $1 / \gamma$, respectively. This shows how the probability density function for $\Phi$ approaches a normal distribution in the limit of large $\gamma$. This transition to normal distributed fluctuations is expected from the central limit theorem, since in this case a large number of uncorrelated pulses contribute to $\Phi(t)$ at any given time. This normal limit is valid for arbitrary pulse shapes and amplitude distributions as far as the cumulants are finite. 


\section{B. Strong intermittency limit}

The asymptotic probability density function in the strong intermittency limit $\gamma \rightarrow 0$ can be obtained for an exponential pulse shape and exponentially distributed pulse amplitudes by neglecting overlap of individual pulse events. Considering first a single pulse, given by $\phi(t)=A \Theta(t) \exp \left(-t / \tau_{\mathrm{d}}\right)$, the time $\mathrm{d} t$ spent between $\phi$ and $\phi+\mathrm{d} \phi$ is given by

$$
\frac{\mathrm{d} t}{\mathrm{~d} \phi}=\frac{\tau_{\mathrm{d}}}{\phi}
$$

Note that due to the assumed exponential pulse shape, the pulse amplitude $A$ does not enter this expression. The number of pulses with amplitude above $\Phi$ is given by the complementary cumulative amplitude distribution function, $\exp (-\Phi /\langle A\rangle)$. The probability density function $P_{\Phi}$ is given by the proportion of time which the process $\Phi(t)$ spends in the range from $\Phi$ to $\Phi+\mathrm{d} \Phi$. With the appropriate normalization, the asymptotic probability density function in the strong intermittency limit is thus given by ${ }^{37}$

$$
\lim _{\gamma \rightarrow 0} P_{\Phi}(\Phi)=\lim _{\gamma \rightarrow 0} \frac{1}{\Gamma(\gamma)} \frac{1}{\Phi} \exp \left(-\frac{\Phi}{\langle A\rangle}\right),
$$

where the Gamma function has been introduced

$$
\Gamma(\gamma)=\int_{0}^{\infty} \mathrm{d} \varphi \varphi^{\gamma-1} \exp (-\varphi)
$$

which in the limit of small $\gamma$ is to lowest order given by $1 / \gamma$. The probability density function in Eq. (52) has an exponential tail for large values but is inversely proportional to $\Phi$ for small values due to the long quite period between pulse events in this strong intermittency regime. Different from the normal limit discussed above, this result depends on both the pulse shape and the amplitude distribution, which are both taken to be exponential functions.

\section{Gamma distribution}

For the special case of exponentially distributed pulse amplitudes, the amplitude integral in Eq. (35) is given by $\left\langle A^{n}\right\rangle=n !\langle A\rangle^{n}$, and the factorial thus cancels. Further invoking the exponential pulse shape given in Eq. (9), it follows that the characteristic function for the stationary distribution can be written as

$$
\exp \left[\gamma \sum_{n=1}^{\infty} \frac{(i u\langle A\rangle)^{n}}{n}\right]=(1-i\langle A\rangle u)^{-\gamma} .
$$

This is nothing but the characteristic function for a Gamma distribution with scale parameter $\langle A\rangle$ and shape parameter $\gamma$. Thus, the stationary probability density function for $\Phi$ is given by

$$
P_{\Phi}(\Phi)=\frac{1}{\langle A\rangle \Gamma(\gamma)}\left(\frac{\Phi}{\langle A\rangle}\right)^{\gamma-1} \exp \left(-\frac{\Phi}{\langle A\rangle}\right)
$$

The lowest order moments and asymptotic limits of the Gamma distribution agree with the expressions discussed previously. In particular, the mean value is given by $\langle\Phi\rangle=\gamma\langle A\rangle$ and the variance by $\Phi_{\mathrm{rms}}^{2}=\gamma\langle A\rangle^{2}$. For $\gamma>1$, the most likely amplitude is $(\gamma-1)\langle A\rangle$ and the shape of the distribution function is unimodal and skewed. When $\gamma=1, P_{\Phi}$ becomes an exponential distribution with the mean value given by the average pulse amplitude, $\exp (-\Phi /\langle\Phi\rangle) /\langle\Phi\rangle$. Note that by writing the average pulse amplitude as $\langle A\rangle=\langle\Phi\rangle / \gamma$, the Gamma distribution given in Eq. (55) can be written in terms of the mean value of $\Phi$ as

$$
\langle\Phi\rangle P_{\Phi}(\Phi)=\frac{\gamma}{\Gamma(\gamma)}\left(\frac{\gamma \Phi}{\langle\Phi\rangle}\right)^{\gamma-1} \exp \left(-\frac{\gamma \Phi}{\langle\Phi\rangle}\right),
$$

where the scale parameter is given by $\langle\Phi\rangle / \gamma$ and the shape parameter is given by $\gamma=\langle\Phi\rangle^{2} / \Phi_{\text {rms }}^{2}$. Examples of this normalized Gamma distribution in Eq. (56) is presented in Fig. 2 for various values of $\gamma$.

For large $\gamma$, the probability density function approximates a normal distribution with non-zero mean, as predicted. Introducing the shifted and scaled variable $\tilde{\Phi}$ defined in Eq. (50), the Gamma distribution for $\Phi$ can be written as

$$
\begin{aligned}
P_{\tilde{\Phi}}(\tilde{\Phi})= & \frac{\gamma^{1 / 2+\gamma-1} \exp (-\gamma)}{\Phi_{\mathrm{rms}} \Gamma(\gamma)}\left(\frac{\tilde{\Phi}}{\gamma^{1 / 2}}+1\right)^{-1} \\
& \times\left(\frac{\tilde{\Phi}}{\gamma^{1 / 2}}+1\right) \exp \left(-\gamma^{1 / 2} \tilde{\Phi}\right)
\end{aligned}
$$

Using the asymptotic limits

$$
\begin{gathered}
\lim _{\gamma \rightarrow \infty}\left(\frac{\tilde{\Phi}}{\gamma^{1 / 2}}+1\right)^{-1}=1, \\
\lim _{\gamma \rightarrow \infty} \frac{\Gamma(\gamma)}{(2 \pi)^{1 / 2} \gamma^{1 / 2+\gamma-1} \exp (-\gamma)}=1, \\
\lim _{\gamma \rightarrow \infty}\left(\frac{\tilde{\Phi}}{\gamma^{1 / 2}}+1\right)^{\gamma} \exp \left(-\gamma^{1 / 2} \tilde{\Phi}\right)=\exp \left(-\frac{\tilde{\Phi}^{2}}{2}\right),
\end{gathered}
$$

the normal distribution for $\tilde{\Phi}$ with vanishing mean and unit variance follows directly from Eq. (57), although formally, the Gamma distribution is defined only for positive values of

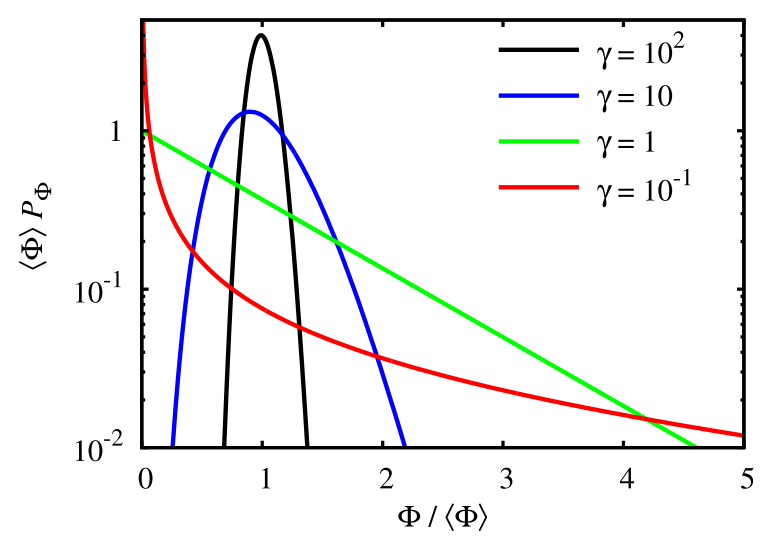

FIG. 2. Normalized Gamma distribution for the random variable $\Phi$ with mean $\langle\Phi\rangle$ for various shape parameters $\gamma$. 
$\Phi$, while the normal distribution is defined for all values of $\tilde{\Phi}$. The Gamma distribution is thus in agreement with the general result stated by Eq. (49).

\section{LEVEL CROSSINGS AND EXCESS TIMES}

In order to further analyze the intermittent features of the process, level crossings and excess time statistics are investigated, in particular, the average duration of time intervals where the signal exceeds some prescribed threshold level.

The fraction of time a signal $\Phi(t)$ spends below a particular threshold value is given by the cumulative distribution function, $C_{\Phi}$, defined in terms of the probability density function $P_{\Phi}(\Phi)$ by

$$
C_{\Phi}(\Phi)=\int_{-\infty}^{\Phi} \mathrm{d} \Phi^{\prime} P_{\Phi}\left(\Phi^{\prime}\right)
$$

Similarly, the fraction of time the signal $\Phi(t)$ spends above a particular threshold value is given by the complementary cumulative distribution function, $1-C_{\Phi}$. This is also known as the survival or reliability function, which is a property of any random variable that maps a set of events, usually associated with mortality or failure of some system, onto time. It captures the probability that the system will survive beyond a specified time.

For the stochastic process considered here with an exponential pulse shape and exponentially distributed pulse amplitudes, $P_{\Phi}$ is given by the Gamma distribution in Eq. (56), and the cumulative distribution function is the regularized lower incomplete Gamma function

$$
C_{\Phi}(\Phi)=\frac{\Gamma_{l}(\gamma, \gamma \Phi /\langle\Phi\rangle)}{\Gamma(\gamma)}
$$

where $\Gamma_{l}$ is the lower incomplete Gamma function

$$
\Gamma_{l}(\gamma, \gamma \Phi /\langle\Phi\rangle)=\int_{0}^{\gamma \Phi /\langle\Phi\rangle} \mathrm{d} \Psi \Psi^{\gamma-1} \exp (-\Psi)
$$

The complementary cumulative distribution function is presented in Fig. 3 for various values of the intermittency parameter $\gamma$. For low values of $\Phi$, the fraction of time above

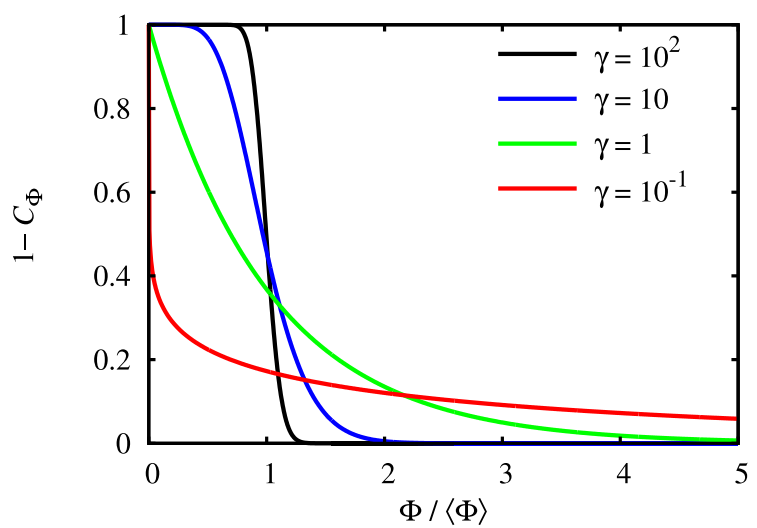

FIG. 3. Complementary cumulative distribution function for the stochastic process for various intermittency parameters. the threshold approaches unity since the entire signal ends up above the threshold. In the normal limit, there are only small variations around the mean value, and the complementary cumulative distribution approaches a step function. Clearly, the fraction of time spent above large threshold levels increases drastically as the intermittency parameter $\gamma$ decreases. In the strong intermittency limit, virtually every new pulse arrival takes the signal above a finite threshold value.

In order to quantify the excess time statistics, the number of up-crossings $X_{\Phi}$ of the level $\Phi$ in an interval of duration $T$ for the process described by Eq. (6) must be derived. For the exponential pulse shape given in Eq. (9), this can be calculated by means of a change of variables formula in the framework of functions of bounded variation. This gives an explicit formula for the Fourier transform of the level crossings function of the filtered Poisson process with jumps. In the case of exponentially distributed pulse amplitudes, the average frequency of up-crossings is found to be given by $39,56,57$

$$
\frac{\tau_{\mathrm{d}}}{T} X_{\Phi}(\Phi)=\frac{1}{\Gamma(\gamma)}\left(\frac{\gamma \Phi}{\langle\Phi\rangle}\right)^{\gamma} \exp \left(-\frac{\gamma \Phi}{\langle\Phi\rangle}\right) .
$$

This function is presented in Fig. 4 for various values of $\gamma$. The number of crossings is evidently proportional to the length of the time series $T$ and inversely proportional to the pulse duration $\tau_{\mathrm{d}}$. In the normal limit, $\gamma \rightarrow \infty$, there are no crossings for levels much smaller or much larger than the mean value due to the absence of large-amplitude fluctuations. The rate of level crossings is therefore a narrow Gaussian function in this limit. Indeed, the relevant limit is straightforward to calculate and gives

$$
\lim _{\gamma \rightarrow \infty} \frac{\tau_{\mathrm{d}}}{T \gamma^{1 / 2}} X_{\tilde{\Phi}}(\tilde{\Phi})=\frac{1}{(2 \pi)^{1 / 2}} \exp \left(-\frac{\tilde{\Phi}^{2}}{2}\right),
$$

where $\tilde{\Phi}=(\Phi-\langle\Phi\rangle) / \Phi_{\text {rms }}$. In the strong intermittency limit, $\gamma \rightarrow 0$, the signal spends most of the time close to zero value, and virtually any pulse arrival will give rise to a level crossing for finite threshold values. As seen in Fig. 4, the rate of level crossings approaches a step function in this limit.

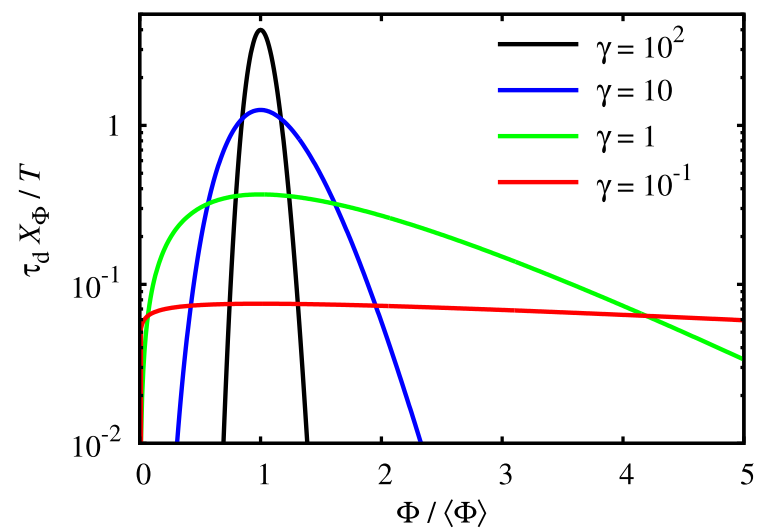

FIG. 4. Frequency of up-crossings of the threshold level $\Phi$ for the stochastic process for various intermittency parameters. 
The average time spent above the threshold $\Phi$ is estimated by the ratio of the average total time above $\Phi$, given by the cumulative distribution, and the average number of up-crossings

$$
\frac{1}{\tau_{\mathrm{d}}}\left\langle\Delta T_{\uparrow}\right\rangle=\frac{1-C_{\Phi}(\Phi)}{\tau_{\mathrm{d}} X_{\Phi}(\Phi) / T}=\frac{\Gamma_{u}(\gamma, \gamma \Phi /\langle\Phi\rangle) \exp (\gamma \Phi /\langle\Phi\rangle)}{(\gamma \Phi /\langle\Phi\rangle)^{\gamma}},
$$

where the upper incomplete Gamma function is given by $\Gamma_{u}(\gamma, \gamma \Phi /\langle\Phi\rangle)=\Gamma(\gamma)-\Gamma_{l}(\gamma, \gamma \Phi /\langle\Phi\rangle)$, using Eq. (61). The average time spent above the threshold $\Phi$ is presented in Fig. 5 for various values of $\gamma$. In all cases, the average excess time decreases monotonically with the threshold level, with a fast drop for small threshold values. This is followed by a slow tapering off for large threshold values. For the range of intermittency parameters considered here, the average excess time is of the order of the pulse duration or shorter for large threshold values. In the limit of large threshold values, the average excess time is given by

$$
\lim _{\Phi /\langle\Phi\rangle \rightarrow \infty} \frac{1}{\tau_{\mathrm{d}}}\left\langle\Delta T_{\uparrow}\right\rangle=\lim _{\Phi /\langle\Phi\rangle \rightarrow \infty} \frac{\langle\Phi\rangle}{\gamma \Phi},
$$

that is, the excess time is inversely proportional to both the intermittency parameter and the threshold value normalized to the mean.

In addition to excess times, the average time spent below a given threshold value, $\left\langle\Delta T_{\downarrow}\right\rangle$, is also readily estimated by the cumulative distribution and the average number of level crossings

$$
\frac{1}{\tau_{\mathrm{d}}}\left\langle\Delta T_{\downarrow}\right\rangle=\frac{C_{\Phi}(\Phi)}{\tau_{\mathrm{d}} X_{\Phi}(\Phi) / T}=\frac{\Gamma_{l}(\gamma, \gamma \Phi /\langle\Phi\rangle) \exp (\gamma \Phi /\langle\Phi\rangle)}{(\gamma \Phi /\langle\Phi\rangle)^{\gamma}} .
$$

This function is presented in Fig. 6 for various values of $\gamma$. As expected, in the normal limit, the signal spends very short times below small values and very long times below large values, resulting in a sharp variation around $\Phi /\langle\Phi\rangle$. With increasing $\gamma$, this variation with the threshold level becomes gradually weaker, and in the strong intermittency regime, there is a weak dependence on the threshold level with a slow increase of the average time below threshold with increasing $\Phi$.

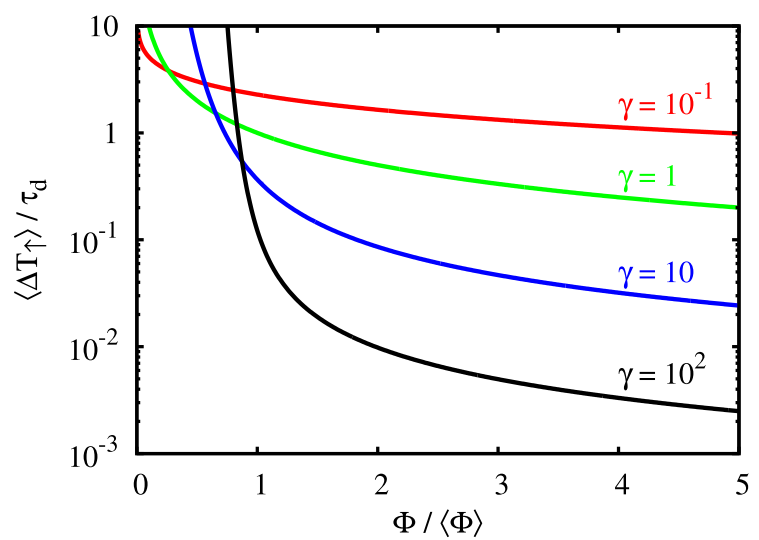

FIG. 5. Average duration of time intervals above the threshold level $\Phi$ for the stochastic process for various intermittency parameters.

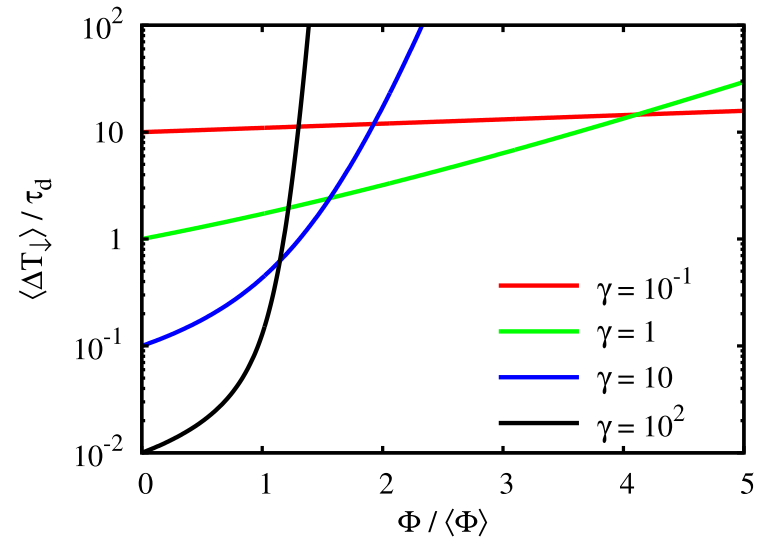

FIG. 6. Average duration of time intervals below the threshold level $\Phi$ for the stochastic process for various intermittency parameters.

\section{MOMENT ESTIMATION}

For the stochastic process described here, the mean squared error on estimates of sample mean and variance can be derived. The mean squared error on an estimator measures the difference between the estimator and what is estimated through the second moment with respect to the origin of the error. For an unbiased estimator, the mean squared error is the variance of the estimator. The mean squared error for the estimated mean value and variance of the process is analyzed in this section.

\section{A. Sample mean}

Consider a realization of the stochastic process given by Eq. (6) sampled at $N$ equi-distant times, $\Phi\left(t_{n}\right)$, where $t_{n}=n \Delta_{t}$, $\Delta_{t}=T / N$ is the sampling time and $n$ is an integer ranging from 1 to $N$. The unbiased estimator of the sample mean of the signal is given by

$$
\hat{\mu}_{\Phi}=\frac{1}{N} \sum_{n=1}^{N} \Phi\left(t_{n}\right)
$$

For an unbiased estimator the relation $\left\langle\hat{\mu}_{\Phi}\right\rangle=\langle\Phi\rangle$ holds, and the mean squared error for the mean of the signal is given by

$$
\begin{aligned}
\operatorname{MSE}\left(\hat{\mu}_{\Phi}\right) & =\left\langle\left(\hat{\mu}_{\Phi}-\langle\Phi\rangle\right)^{2}\right\rangle \\
& =-\langle\Phi\rangle^{2}+\frac{1}{N^{2}} \sum_{n=1}^{N} \sum_{m=1}^{N}\left\langle\Phi\left(t_{n}\right) \Phi\left(t_{m}\right)\right\rangle
\end{aligned}
$$

In order to evaluate the sum over the discrete correlation function, the continuous two-point correlation function given by Eq. (28) at the discrete sampling times is used. For an exponential pulse shape, this gives

$$
\operatorname{MSE}\left(\hat{\mu}_{\Phi}\right)=\frac{\Phi_{\mathrm{rms}}^{2}}{N}\left[1+\frac{1}{N} \sum_{\substack{n, m=1 \\ n \neq m}}^{N} \exp \left(-|n-m| \frac{\Delta_{t}}{\tau_{\mathrm{d}}}\right)\right],
$$

where the first term inside the square brackets follows from the $N$ terms in Eq. (68) for which $n=m$. Defining $\theta=\Delta_{t} / \tau_{\mathrm{d}}$ 
as the normalized sampling time, the sum in the above equation is evaluated as a geometric series to give the mean squared error on the estimate of the mean value of the random variable

$\operatorname{MSE}\left(\hat{\mu}_{\Phi}\right)=\frac{\Phi_{\mathrm{rms}}^{2}}{N}\left[1+\frac{1}{N} \frac{N+\exp (-\theta N)-1-N \exp (-\theta)}{2 \sinh ^{2}(\theta / 2)}\right]$.

As expected, the dominant contribution to the mean squared error is inversely proportional to the number of samples $N$. For the process considered here, the relative mean squared error, $\operatorname{MSE}\left(\hat{\mu}_{\Phi}\right) /\langle\Phi\rangle^{2}$, is inversely proportional to $\gamma$. Thus, a strongly intermittent process, $\gamma \ll 1$, features a much larger relative error on estimates of the mean than a process with significant pulse overlap, $\gamma \gg 1$, given the same number of samples and sampling rate. The mean squared error as a function of the sample size is presented in Fig. 7 for various normalized sampling rates.

For $\theta \gg 1$, the obtained samples are uncorrelated and the expression for the mean squared error simplifies by expansion of the hyperbolic function to

$$
\lim _{\theta \rightarrow \infty} \operatorname{MSE}\left(\hat{\mu}_{\Phi}\right)=\frac{\Phi_{\mathrm{rms}}^{2}}{N},
$$

which is the same as the well-known result for uncorrelated and normal distributed samples. The mean squared error in this limit can alternatively be written as $\operatorname{MSE}\left(\hat{\mu}_{\Phi}\right)=\langle\Phi\rangle^{2} / \gamma N$, again showing that the relative error for the estimated mean is large in the strong intermittency limit. The opposite limit $\theta \ll 1$ describes the case of high sampling frequency where the time series is well resolved on the temporal scale of the individual pulses. In this case, the mean squared error simplifies to

$$
\lim _{\theta \rightarrow 0} \operatorname{MSE}\left(\hat{\mu}_{\Phi}\right)=\lim _{\theta \rightarrow 0} \frac{\Phi_{\mathrm{rms}}^{2}}{N}\left[1+\frac{2}{N} \frac{\exp (-\theta N)-(1-\theta N)}{\theta^{2}}\right] .
$$

Further taking the limit $\theta N \gg 1$, describing the case of a total sample time long compared to the pulse duration time, $T=N \Delta_{t} \gg \tau_{\mathrm{d}}$, the mean squared error on the mean is to the lowest order given by

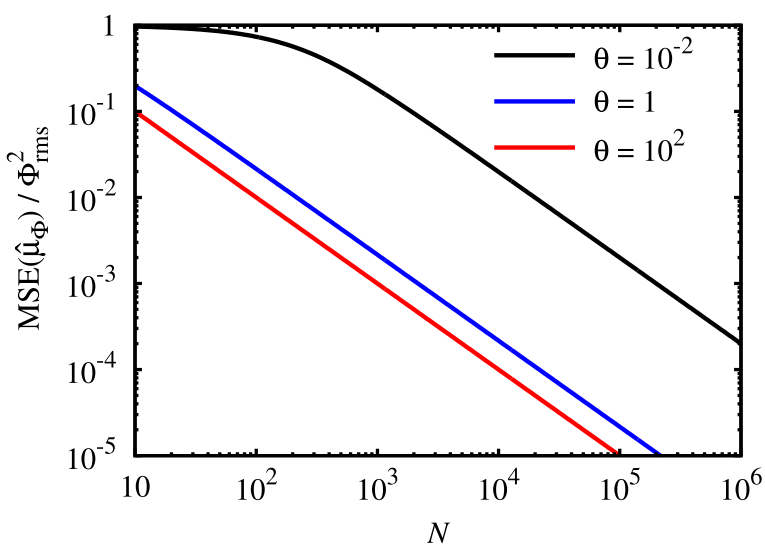

FIG. 7. Mean squared error on the sample estimate of the mean value of a realization of the stochastic process for various normalized sampling rates.

$$
\lim _{\substack{\theta \rightarrow 0 \\ \theta N \rightarrow \infty}} \operatorname{MSE}\left(\hat{\mu}_{\Phi}\right)=\lim _{\theta N \rightarrow \infty} \frac{2 \Phi_{\mathrm{rms}}^{2}}{\theta N} .
$$

This clearly shows how finite correlation effects between the samples contribute by an amplification factor $2 / \theta$ to the mean squared error on $\hat{\mu}_{\Phi}$ for a fixed number of samples. Alternatively, the relative mean squared error in this limit can be written as $\operatorname{MSE}\left(\hat{\mu}_{\Phi}\right) /\langle\Phi\rangle^{2}=2 \tau_{\mathrm{w}} / T$, showing that a long sampling time relative to the average pulse waiting time reduces the relative mean squared error in the case of high sampling frequency.

Instead of evaluating the geometrical sum that leads to Eq. (70), the sum over the two-point correlation function can be written as a Riemann sum and approximated by an integral

$$
\begin{aligned}
\lim _{\theta \rightarrow 0} & \sum_{\substack{n, m=1 \\
n \neq m}}^{N} \exp (-\theta|n-m|) \\
= & \lim _{\theta \rightarrow 0} \int_{0}^{N} \mathrm{~d} n \int_{0}^{N} \mathrm{~d} m[\Theta(n-m) \exp [\theta(m-n)] \\
& +\Theta(m-n) \exp [\theta(n-m)]] \\
= & \lim _{\theta \rightarrow 0} 2 \frac{\theta N+\exp (-\theta N)-1}{\theta^{2}}
\end{aligned}
$$

This gives the same result as in Eq. (72). For this approximation to be valid, the variation of the integrand must be small, $\mathrm{d} n / N \ll 1$ and $\mathrm{d} m / N \ll 1$, which corresponds to the high sampling frequency limit $\theta \ll 1$.

\section{B. Sample variance}

An expression for the mean squared error on the unbiased estimator for the variance,

$$
\hat{\sigma}_{\Phi}^{2}=\frac{1}{N-1} \sum_{n=1}^{N}\left[\Phi\left(t_{n}\right)-\langle\Phi\rangle\right]^{2}
$$

may be derived using the same approach as used to obtain Eq. (70). The mean squared error for this unbiased estimator of the sample variance is given by

$$
\operatorname{MSE}\left(\hat{\sigma}_{\Phi}^{2}\right)=\left\langle\left(\hat{\sigma}_{\Phi}^{2}-\Phi_{\mathrm{rms}}^{2}\right)^{2}\right\rangle .
$$

As is clear from the above equation, calculation of the mean squared error of the variance involves correlation functions for the fourth power of the signal $\Phi(t)$. While this can in principle be calculated analytically, the resulting expressions are lengthy and do not provide much insight. However, the relevant asymptotic limits are readily obtained for high and low sampling rates as discussed above for the mean. In the case of low sampling frequency, $\theta \gg 1$, the obtained samples are independent and identically distributed, and the mean squared error for the sample variance is given by ${ }^{58}$

$$
\lim _{\theta \rightarrow \infty} \operatorname{MSE}\left(\hat{\sigma}_{\Phi}^{2}\right)=\frac{\Phi_{\mathrm{rms}}^{4}}{N}\left(\frac{2 N}{N-1}+\frac{1}{\gamma} \frac{\left\langle A^{4}\right\rangle}{\left\langle A^{2}\right\rangle^{2}}\right),
$$


where the last term within the parenthesis is the excess flatness for the stochastic process. For exponentially distributed pulse amplitudes, this is given by $6 / \gamma$, revealing large errors on the estimator in the strong intermittency limit.
In the case of high sampling rate, $\theta \ll 1$, calculation of quadruple sums in Eq. (76) can be approximated by integrals as in Eq. (74). A lengthy calculation gives the unbiased mean squared error on the variance in the limit $\theta \rightarrow 0$

$$
\begin{aligned}
& \frac{2 \Phi_{\mathrm{rms}}^{4}}{\alpha N}\left(1+\frac{3}{\gamma}\right)+\frac{\Phi_{\mathrm{rms}}^{4}}{\theta^{2} N^{2}}[-5-8 \exp (-\theta N)+\exp (-2 \theta N)] \\
& \quad+\frac{3 \Phi_{\mathrm{rms}}^{4}}{\gamma \theta^{2} N^{2}}[\exp (-2 \theta N)-9]+\frac{4 \Phi_{\mathrm{rms}}^{4}}{\theta^{3} N^{3}}[1-2 \exp (-\theta N)+\exp (-2 \theta N)] \\
& \quad+\frac{4 \Phi_{\mathrm{rms}}^{4}}{\gamma \theta^{3} N^{3}}[14-9 \exp (-\theta N)+\exp (-3 \theta N)]+\frac{12 \Phi_{\mathrm{rms}}^{4}}{\theta^{4} N^{4}}[1-2 \exp (-\theta N)+\exp (-2 \theta N)] \\
& \quad+\frac{4 \Phi_{\mathrm{rms}}^{4}}{\gamma \theta^{4} N^{4}}[-11+18 \exp (-\theta N)-9 \exp (-2 \theta N)+2 \exp (-3 \theta N)],
\end{aligned}
$$

here given for the case of exponentially distributed pulse amplitudes. The mean squared error on the variance as a function of the sample size is presented in Fig. 8 for various values of the intermittency parameter $\gamma$.

In the limit of large sample numbers or long record length, $\theta N=T / \tau_{\mathrm{d}} \gg 1$, the leading order terms in Eq. (78) are inversely proportional to $\theta N$, giving the mean squared error on the variance

$$
\lim _{\substack{\theta \rightarrow 0 \\ \theta N \rightarrow \infty}} \operatorname{MSE}\left(\hat{\sigma}_{\Phi}^{2}\right)=\lim _{\theta N \rightarrow \infty} \frac{\Phi_{\mathrm{rms}}^{4}}{\theta N}\left(2+\frac{6}{\gamma}\right) .
$$

In the normal limit, $\gamma \gg 1$, the mean squared error simplifies to $\operatorname{MSE}\left(\hat{\sigma}_{\Phi}^{2}\right)=2 \tau_{\mathrm{d}} \Phi_{\mathrm{rms}}^{4} / T N$, analogous to the error on estimates of the mean value of the signal given in Eq. (73). In the strong intermittency regime, $\gamma \ll 1$, the mean squared error can be written as $\operatorname{MSE}\left(\hat{\sigma}_{\Phi}^{2}\right)=6 \tau_{\mathrm{w}} \Phi_{\mathrm{rms}}^{4} / T N$, again requiring the total sample time to be much longer than the average pulse waiting time for small relative errors. The mean squared error on variance for $\theta \ll 1$ and errors on estimates of sample skewness and flatness moments have been discussed and investigated by use of synthetic data in Ref. 38 .

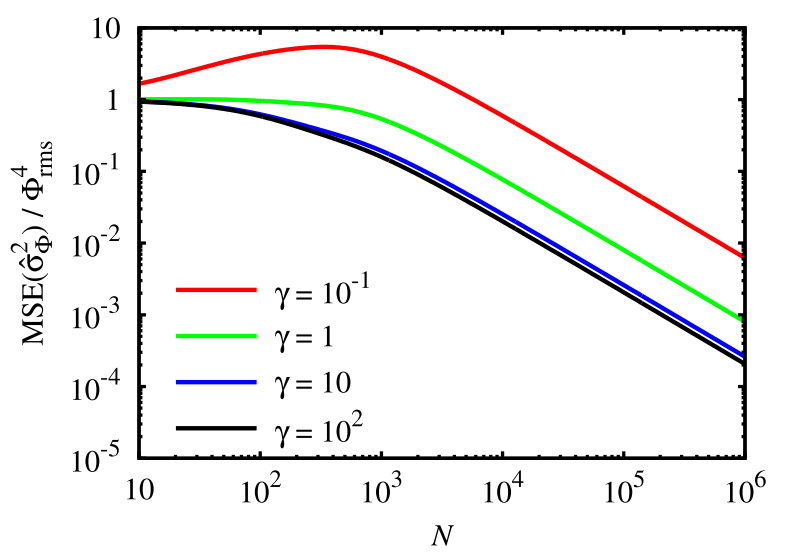

FIG. 8. Mean squared error on the sample estimate of the variance of a realization of the stochastic process for $\theta=10^{-2}$ and various intermittency parameters $\gamma$.

\section{DISCUSSION}

The results above are discussed in the context of filament motion in magnetically confined plasmas and broad average profiles. This is followed by a summary of the underlying assumptions and predictions of the stochastic model and comparison to recent experimental measurements.

\section{A. Exponential mean profile}

Returning to the discussion of pulse propagation in Sec. II A, the plasma density is modelled by a super-position of pulses which is given by

$$
\Phi_{K}(x, t)=\sum_{k=1}^{K} \phi_{k}(x, t) .
$$

Neglecting interaction between pulse structures, their evolution is governed by the modified advection equation,

$$
\frac{\partial \phi_{k}}{\partial t}+v_{k} \frac{\partial \phi_{k}}{\partial x}+\frac{\phi_{k}}{\tau_{\|}}=0
$$

where $v_{k}$ is the radial velocity for pulse $k$ and the last term on the left hand side describes B-parallel losses due to acoustic streaming along the field lines. In the SOL, the parallel transit time $\tau_{\|}$is typically estimated by $L_{\|} / C_{\mathrm{S}}$, where $L_{\|}$is the magnetic connection length and $C_{\mathrm{s}}$ is the acoustic speed. ${ }^{59-61}$ According to Eq. (81), individual pulse structures are thus described by

$$
\phi_{k}(x, t)=A_{k}(t) \varphi_{k}\left(x-x_{k}-v_{k} t\right),
$$

where $\varphi_{k}(x)$ describes the pulse shape. At time $t=0$, the pulse labeled $k$ is located at the reference position $x_{k}$. The amplitude $A_{k}$ for event $k$ now varies with time and satisfies the equation

$$
\frac{\mathrm{d} A_{k}}{\mathrm{~d} t}=-\frac{A_{k}}{\tau_{\|}} .
$$

Introducing the pulse amplitude $A_{0 k}$ for the pulse labeled $k$ at position $x=0$, the solution of the amplitude equation can be written as 


$$
A_{k}(t)=A_{0 k} \exp \left(-\frac{t+x_{k} / v_{k}}{\tau_{\|}}\right)
$$

Thus, at time $-x_{k} / v_{k}$ the pulse labeled $k$ is located at $x=0$ and the amplitude is $A_{0 k}$. Consider now the signal recorded at a reference position $\xi$. Introducing the reference time $t_{k}$ $=\left(\xi-x_{k}\right) / v_{k}$ for pulse $k$ to be at the position $\xi$, the signal can be written as

$$
\begin{aligned}
\Phi_{K}(\xi, t)= & \sum_{k=1}^{K} A_{0 k} \exp \left(-\frac{\xi}{v_{k} \tau_{\|}}\right) \\
& \times \exp \left(-\frac{t-t_{k}}{\tau_{\|}}\right) \varphi_{k}\left(-v_{k}\left(t-t_{k}\right)\right) .
\end{aligned}
$$

Clearly, the average profile decreases exponentially with a scale length that increases with increasing pulse velocities and parallel loss time.

In the particular case of an exponential pulse shape,

$$
\varphi_{k}(x)=\Theta\left(-\frac{x}{\ell_{k}}\right) \exp \left(\frac{x}{\ell_{k}}\right)
$$

the signal recorded at the reference position $\xi$ is given by

$$
\Phi_{K}(\xi, t)=\sum_{k=1}^{K} A_{0 k} \exp \left(-\frac{\xi}{v_{k} \tau_{\|}}\right) \Theta\left(\frac{t-t_{k}}{\tau_{\perp}}\right) \exp \left(-\frac{t-t_{k}}{\tau_{\mathrm{d}}}\right)
$$

where $\tau_{\perp}=\ell_{k} / v_{k}$ and the pulse duration is now given by the harmonic mean of the perpendicular and parallel transit times, $\tau_{\mathrm{d}}=\tau_{\|} \tau_{\perp} /\left(\tau_{\|}+\tau_{\perp}\right)$. As expected, the duration of a pulse recorded at position $\xi$ will be influenced by both perpendicular and parallel transport. In the absence of parallel losses, the expression (87) reduces to Eq. (5).

For any fixed position $\xi$, Eq. (87) is just the process that has been analysed in the present contribution. Assuming for simplicity the same size $\ell_{\perp}$ and the velocity $v_{\perp}$ for all pulses so that the pulse duration is constant, the mean value of the process gives the profile

$$
\langle\Phi\rangle(\xi)=\frac{\tau_{\mathrm{d}}}{\tau_{\mathrm{w}}}\left\langle A_{0}\right\rangle \exp \left(-\frac{\xi}{v_{\perp} \tau_{\|}}\right) .
$$

This equation elucidates the importance of pulse size, amplitude, velocity, and waiting time for broad profiles and high average plasma densities in the SOL. In particular, the mean value is proportional to the pulse duration and mean amplitude and inversely proportional to the average waiting time. Moreover, the profile scale length is proportional to the pulse velocity and parallel transit time, as often assumed in heuristic modelling of convective cross-field transport in the SOL. ${ }^{60-64}$

\section{B. Comparison with experiments}

Intermittent fluctuations in the boundary region of magnetically confined plasmas have been modeled by a superposition of uncorrelated pulses with fixed shape and duration.
For this stochastic process, the model parameters are the pulse duration $\tau_{\mathrm{d}}$, the average waiting time $\tau_{\mathrm{w}}$, and the average pulse amplitude $\langle A\rangle$. The lowest order moments of the random variable have been derived for general pulse shapes and amplitude distributions. The mean value of the random variable is given by the average pulse amplitude and the ratio of the pulse duration and average waiting time, while there is a universal parabolic relation between the skewness and flatness moments. Such a relation has been reported from many experimental investigations of intermittent fluctuations in the plasma boundary region. ${ }^{11-14,40-42}$

In the particular case of an exponential pulse shape, the auto-correlation function is shown to be given by an exponential function with a shape that is independent of the average pulse waiting time, again in agreement with experimental measurements. ${ }^{13,15}$ Using this two-point correlation, the mean squared errors on sample mean and variance are derived and their dependence on model parameters and the sampling rate are elucidated. These results are particularly useful for estimating the errors involved when calculating the radial profile of the mean plasma density and its fluctuation level from measurements in the boundary region of hot fusion plasmas. In normal operation, reciprocating Langmuir probes move radially through the SOL up to the last closed magnetic flux surface to record radial profiles and fluctuations of the ion saturation current and floating potential..$^{20-22,27-30,32,40,41}$ In order to prevent arcing and damage of the probe head during measurements in hot plasmas, the probe moves rapidly through the boundary region. For any given radial position, this yields short data time series with corresponding limitations and uncertainties in the calculation of statistical averages. For example, consider the typical case for many tokamak plasmas with a sampling time of $0.5 \mu \mathrm{s}$, time series duration of $5 \mathrm{~ms}$, pulse duration $\tau_{\mathrm{d}}=15 \mu \mathrm{s}$, and a relative fluctuation level $\Phi_{\mathrm{rms}} /\langle\Phi\rangle=0.5$. This gives a relative error on estimates of the mean values of the signal of $5 \%$ and a relative error on estimates of the standard deviation of $10 \%$.

In order to elucidate the statistical properties of plasma fluctuations in the SOL, dedicated experiments were performed on several confinement devices with the probe maintained at a fixed spatial position at the outboard mid-plane in ohmically heated, lower single null, deuterium fuelled plasmas to record very long time series under stationary plasma conditions. Analysis of such measurement data, as well as long data time series from gas puff imaging, provides strong evidence in support of the stochastic model presented here. ${ }^{11-15}$ Just in front of the main chamber wall at the outboard mid-plane region, large relative fluctuation levels are measured, corresponding to an intermittency parameter $\gamma$ of order unity. Using conditional averaging, it has been shown that plasma density time series are dominated by largeamplitude bursts attributed to blob-like filament structures moving through the SOL. The average burst shape is well described by an exponential wave-form with constant duration, while the waiting times and peak amplitudes of the bursts both have an exponential distribution. ${ }^{11-15}$

When the pulse amplitudes are exponentially distributed, the probability density function for the random variable is a Gamma distribution with the shape parameter given by 
the ratio of the pulse duration and average waiting time and the scale parameter given by the average pulse amplitude. In the case of significant pulse overlap, $\gamma \gg 1$, the probability density function approaches a normal distribution, as expected from the central limit theorem, independent of the pulse shape and amplitude distribution. ${ }^{35}$ It should be noted that when comparing the predicted Gamma distribution to experimental data, there are no free fit parameters since the shape parameter is given by the sample mean and variance, $\gamma=\langle\Phi\rangle^{2} / \Phi_{\text {rms }}^{2}$. There are many reports from experimental measurements revealing histograms of plasma fluctuations that are well described by a Gamma distribution across a large range of experimental control parameters and locations in the SOL. ${ }^{11-15,40,41}$

By taking into account the loss of plasma along magnetic field lines in the SOL, it is here demonstrated that an exponential mean plasma density profile is expected for the simple case of constant pulse size and velocity. The profile scale length is then given by the product of the radial pulse velocity and the parallel transit time. From experiments on magnetically confined plasmas, it is commonly found that the average profile is indeed exponential in the far SOL with a scale length which increases with increasing line-averaged density. ${ }^{17-29,32,33}$ It has also been found that this profile broadening is accompanied by larger average radial blob velocities. $29,32,33,65$ The stochastic model presented here clearly reveals how such an increase in the pulse velocity leads to broader profiles in the SOL. Experiments also show profile broadening with decreasing plasma current, which is also associated with larger collisionality and blob velocities. ${ }^{17,29}$ The profile broadening due to radial motion of blob-like structures described here is possibly linked to divertor detachment and the empirical discharge density limit. $^{20,21,29,32,33}$

For any given realization of the process, or its possible description of an experimental data time series, the model parameters $\tau_{\mathrm{d}}, \tau_{\mathrm{w}}$, and $\langle A\rangle$ are not directly accessible. However, the predictions of the model readily allow to estimate them. The auto-correlation function in Eq. (28) can be used to obtain the pulse duration, and the sample mean and variance can be used to obtain the intermittency parameter $\gamma$ and average pulse amplitude. The results presented in Sec. VI describe the errors involved in estimating the sample mean and variance and thereby the model parameters. This provides a novel approach for investigating measurement data and elucidating systematic variations with experimental control parameters.

\section{CONCLUSIONS}

A stochastic model for intermittent fluctuations in the plasma boundary region has been constructed by a superposition of uncorrelated pulses which represent radial motion of blob-like structures. Both the underlying assumptions and the predictions of this model are consistent with recent measurements in magnetically confined plasmas. The mean amplitude of the signal is given by the average pulse amplitude times the ratio of pulse duration and average waiting time. In the case of exponentially distributed pulse amplitudes, the probability density function of the plasma density is shown to be a Gamma distribution with the shape parameter determined by the degree of pulse overlap. This simple model thus explains the salient fluctuation statistics found in numerous experimental measurements and elucidates the role of burst statistics for large SOL plasma densities and fluctuation levels.

The stochastic model suggests a new approach to study the role of fluctuations in the boundary region of fusion plasmas by revealing the dependence of the intermittency factor $\gamma$ on plasma parameters. Universal properties and scaling relationships can be identified by comparison of data from various experimental devices and across a broad range of plasma parameters. Moreover, the use of level crossing rates and excess time statistics is a novel approach to describe intermittency effects that promises to be particularly relevant for plasma-wall interaction in hot fusion plasmas. Novel predictions for these statistics have been derived in the present contribution, extending the well-known normal limit previously compared to measurements data in basic laboratory and space plasmas. ${ }^{48,49}$

Indications are that the results obtained in the present study can apply for some seemingly universal features of fluctuations found in magnetized plasmas. The data discussed in Sec. VII originated from hot toroidal plasmas, but reports from experiments in low temperature linear devices show raw data that can also be interpreted as a random super-position of pulses with an exponential form in some additive random noise. ${ }^{66,67}$ In the light of these results, it is worthwhile to analyze the data also from other types of experiments in magnetized plasmas. More generally, the theory presented here can be seen as a reference model for intermittent fluctuations and is well known to be relevant for many other applications beyond fusion plasmas. $48,49,56,57$

\section{ACKNOWLEDGMENTS}

This work was supported with financial subvention from the Research Council of Norway under Grant No. 240510/F20.

${ }^{1}$ D. A. D'Ippolito, J. R. Myra, S. I. Krasheninnikov, G. Q. Yu, and A. Yu. Pigarov, Contrib. Plasma Phys. 44, 205 (2004).

${ }^{2}$ S. J. Zweben, J. A. Boedo, O. Grulke, C. Hidalgo, B. LaBombard, R. J. Maqueda, P. Scarin, and J. L. Terry, Plasma Phys. Controlled Fusion 49, S1 (2007).

${ }^{3}$ S. I. Krasheninnikov, D. A. D’Ippolito, and J. R. Myra, J. Plasma Phys. 74, 679 (2008).

${ }^{4}$ O. E. Garcia, Plasma Fusion Res. 4, 19 (2009).

${ }^{5}$ D. A. D'Ippolito, J. R. Myra, and S. J. Zweben, Phys. Plasmas 18, 060501 (2011).

${ }^{6}$ J. L. Terry, N. P. Basse, I. Cziegler, M. Greenwald, O. Grulke, B. LaBombard, S. J. Zweben, E. M. Edlund, J. W. Hughes, L. Lin, Y. Lin, M. Porkolab, M. Sampsell, B. Veto, and S. J. Wukitch, Nucl. Fusion 45, 1321 (2005).

${ }^{7}$ O. Grulke, J. L. Terry, B. LaBombard, and S. J. Zweben, Phys. Plasmas 13, 012306 (2006).

${ }^{8}$ S. J. Zweben, R. J. Maqueda, J. L. Terry, T. Munsat, J. R. Myra, D. DIppolito, D. A. Russell, J. A. Krommes, B. LeBlanc, T. Stoltzfus-Dueck, D. P. Stotler, K. M. Williams, C. E. Bush, R. Maingi, O. Grulke, S. A. Sabbagh, and A. E. White, Phys. Plasmas 13, 056114 (2006).

${ }^{9}$ R. Kube, O. E. Garcia, B. LaBombard, J. L. Terry, and S. J. Zweben, J. Nucl. Mater. 438, S505 (2013).

${ }^{10}$ S. J. Zweben, W. M. Davis, S. M. Kaye, J. R. Myra, R. E. Bell, B. P. LeBlanc, R. J. Maqueda, T. Munsat, S. A. Sabbagh, Y. Sechrest, D. P. Stotler, and NSTX Team, Nucl. Fusion 55, 093035 (2015). 
${ }^{11}$ O. E. Garcia, I. Cziegler, R. Kube, B. LaBombard, and J. L. Terry, J. Nucl. Mater. 438, S180 (2013)

${ }^{12}$ O. E. Garcia, S. M. Fritzner, R. Kube, I. Cziegler, B. LaBombard, and J. L. Terry, Phys. Plasmas 20, 055901 (2003).

${ }^{13}$ A. Theodorsen, O. E. Garcia, J. Horacek, R. Kube, and R. A. Pitts, Plasma Phys. Controlled Fusion 58, 044006 (2016).

${ }^{14}$ R. Kube, A. Theodorsen, O. E. Garcia, B. LaBombard, and J. L. Terry, Plasma Phys. Controlled Fusion 58, 054001 (2016).

${ }^{15}$ O. E. Garcia, J. Horacek, and R. A. Pitts, Nucl. Fusion 55, 062002 (2015).

${ }^{16}$ R. A. Pitts, J. P. Coad, D. P. Coster, G. Federici, W. Fundamenski, J. Horacek, K. Krieger, A. Kukushkin, J. Likonen, G. F. Matthews, M. Rubel, J. D. Strachan, and JET-EFDA Contributors, Plasma Phys. Controlled Fusion 47, B303 (2005).

${ }^{17}$ D. L. Rudakov, J. A. Boedo, R. A. Moyer, P. C. Stangeby, J. G. Watkins, D. G. Whyte, L. Zeng, N. H. Brooks, R. P. Doerner, T. E. Evans, M. E. Fenstermacher, M. Groth, E. M. Hollmann, S. I. Krasheninnikov, C. J. Lasnier, A. W. Leonard, M. A. Mahdavi, G. R. McKee, A. G. McLean, A. Yu. Pigarov, W. R. Wampler, G. Wang, W. P. West, and C. P. C. Wong, Nucl. Fusion 45, 1589 (2005).

${ }^{18}$ D. G. Whyte, B. L. Lipschultz, P. C. Stangeby, J. Boedo, D. L. Rudakov, J. G. Watkins, and W. P. West, Plasma Phys. Controlled Fusion 47, 1579 (2005).

${ }^{19}$ B. Lipschultz, X. Bonnin, G. Counsell, A. Kallenbach, A. Kukushkin, K. Krieger, A. Leonard, A. Loarte, R. Neu, R. A. Pitts, T. Rognlien, J. Roth, C. Skinner, J. L. Terry, E. Tsitrone, D. Whyte, S. Zweben, N. Asakura, D. Coster, R. Doerner, R. Dux, G. Federici, M. Fenstermacher, W Fundamenski, P. Ghendrih, A. Herrmann, J. Hu, S. Krasheninnikov, G. Kirnev, A. Kreter, V. Kurnaev, B. LaBombard, S. Lisgo, T. Nakano, N. Ohno, H. D. Pacher, J. Paley, Y. Pan, G. Pautasso, V. Philipps, V. Rohde, D. Rudakov, P. Stangeby, S. Takamura, T. Tanabe, Y. Yang, and S. Zhu, Nucl. Fusion 47, 1189 (2007).

${ }^{20}$ B. LaBombard, R. L. Boivin, M. Greenwald, J. Hughes, B. Lipschultz, D. Mossessian, C. S. Pitcher, J. L. Terry, S. J. Zweben, and Alcator Group, Phys. Plasmas 8, 2107 (2001).

${ }^{21}$ B. LaBombard, J. W. Hughes, D. Mossessian, M. Greenwald, B. Lipschultz, J. L. Terry, and Alcator C-Mod Team, Nucl. Fusion 45, 1658 (2005).

${ }^{22}$ B. LaBombard, J. L. Terry, J. W. Hughes, D. Brunner, J. Payne, M. L. Reinke, I. Cziegler, R. Granetz, M. Greenwald, I. H. Hutchinson, J. Irby, Y. Lin, B. Lipschultz, Y. Ma, E. S. Marmar, W. L. Rowan, N. Tsujii, G. Wallace, D. G. Whyte, S. Wolfe, S. Wukitch, G. Wurden, and Alcator CMod Team, Phys. Plasmas 18, 056104 (2011).

${ }^{23}$ D. L. Rudakov, J. A. Boedo, R. A. Moyer, S. Krasheninnikov, A. W. Leonard, M. A. Mahdavi, G. R. McKee, G. D. Porter, P. C. Stangeby, J. G. Watkins, W. P. West, D. G. Whyte, and G. Antar, Plasma Phys. Controlled Fusion 44, 717 (2002).

${ }^{24}$ G. Y. Antar, G. Counsell, Y. Yu, B. Labombard, and P. Devynck, Phys. Plasmas 10, 419 (2003).

${ }^{25}$ J. Horacek, J. Adamek, H. W. Müller, J. Seidl, A. H. Nielsen, V. Rohde, F. Mehlmann, C. Ionita, E. Havlíčková, and ASDEX Upgrade Team, Nucl. Fusion 50, 105001 (2010).

${ }^{26}$ J. A. Boedo, J. R. Myra, S. Zweben, R. Maingi, R. J. Maqueda, V. A. Soukhanovskii, J. W. Ahn, J. Canik, N. Crocker, D. A. D'Ippolito, R. Bell, H. Kugel, B. Leblanc, L. A. Roquemore, D. L. Rudakov, and NSTX Team, Phys. Plasmas 21, 042309 (2014).

${ }^{27}$ O. E. Garcia, R. A. Pitts, J. Horacek, A. H. Nielsen, W. Fundamenski, J. P. Graves, V. Naulin, and J. Juul Rasmussen, Nucl. Fusion 47, 667 (2007).

${ }^{28}$ O. E. Garcia, R. A. Pitts, J. Horacek, A. H. Nielsen, W. Fundamenski, J. P. Graves, V. Naulin, and J. Juul Rasmussen, J. Nucl. Mater. 363-365, 575 (2007).

${ }^{29}$ O. E. Garcia, R. A. Pitts, J. Horacek, J. Madsen, V. Naulin, A. H. Nielsen, and J. Juul Rasmussen, Plasma Phys. Controlled Fusion 49, B47 (2007).

${ }^{30}$ O. E. Garcia, J. Horacek, R. A. Pitts, V. Naulin, A. H. Nielsen, J. Juul Rasmussen, J. Graves, and W. Fundamenski, Plasma Phys. Controlled Fusion 48, L1 (2006).
${ }^{31}$ F. Militello, P. Tamain, W. Fundamenski, A. Kirk, V. Naulin, A. H. Nielsen, and MAST Team, Plasma Phys. Controlled Fusion 55, 025005 (2013).

${ }^{32}$ D. Carralero, G. Birkenmeier, H. W. Müller, P. Manz, P. deMarne, S. H. Müller, F. Reimold, U. Stroth, M. Wischmeier, E. Wolfrum, and ASDEX Upgrade Team, Nucl. Fusion 54, 123005 (2014).

${ }^{33}$ D. Carralero, P. Manz, L. Aho-Mantila, G. Birkenmeier, M. Brix, M. Groth, H. W. Müller, U. Stroth, N. Vianello, E. Wolfrum, ASDEX Upgrade Team, JET Contributors, and EUROfusion MST1 Team, Phys. Rev. Lett. 115, 215002 (2015).

${ }^{34}$ N. Campbell, Proc. Cambridge Philos. Soc. 15, 117 (1909).

${ }^{35}$ S. O. Rice, Bell Syst. Tech. J. 23, 282 (1944).

${ }^{36}$ H. L. Pécseli and J. K. Trulsen, Plasma Phys. Controlled Fusion 35, 1701 (1993).

${ }^{37}$ O. E. Garcia, Phys. Rev. Lett. 108, 265001 (2012).

${ }^{38}$ R. Kube and O. E. Garcia, Phys. Plasmas 22, 012502 (2015).

${ }^{39}$ A. Theodorsen and O. E. Garcia, Phys. Plasmas 23, 040702 (2016).

${ }^{40}$ J. P. Graves, J. Horacek, R. A. Pitts, and K. I. Hopcraft, Plasma Phys. Controlled Fusion 47, L1 (2005).

${ }^{41}$ J. Horacek, R. A. Pitts, and J. P. Graves, Czech. J. Phys. 55, 271 (2005).

${ }^{42}$ B. Labit, I. Furno, A. Fasoli, A. Diallo, S. H. Müller, G. Plyushchev, M. Podestà, and F. M. Poli, Phys. Rev. Lett. 98, 255002 (2007).

${ }^{43}$ F. Sattin, M. Agostini, P. Scarin, N. Vianello, R. Cavazzana, L. Marrelli, G. Serianni, S. J. Zweben, R. J. Maqueda, Y. Yagi, H. Sakakita, H Koguchi, S. Kiyama, Y. Hirano, and J. L. Terry, Plasma Phys. Controlled Fusion 51, 055013 (2009).

${ }^{44}$ A. S. Bergsaker, A. Fredriksen, H. L. Pécseli, and J. K. Trulsen, Phys. Scr. 90, 108005 (2015).

${ }^{45}$ S. O. Rice, Bell Syst. Tech. J. 24, 46 (1945).

${ }^{46}$ R. Barakat, Inf. Sci. 20, 83 (1980).

${ }^{47}$ L. Kristensen, M. Casanova, M. S. Courtney, and I. Troen, Boundary Layer Meteorol. 55, 91 (1991).

${ }^{48}$ H. Sato, H. L. Pécseli, and J. Trulsen, J. Geophys. Res. 117, A03329, doi:10.1029/2011JA017242 (2012).

${ }^{49}$ L. Fattorini, Å. Fredriksen, H. L. Pécseli, C. Riccardi, and J. K. Trulsen, Plasma Phys. Controlled Fusion 54, 085017 (2012).

${ }^{50}$ J. E. Wilkins, Ann. Math. Stat. 15, 333 (1944).

${ }^{51}$ N. Mole and E. D. Clarke, Boundary Layer Meteorol. 73, 35 (1995).

${ }^{52}$ N. Mole, T. P. Schopflocher, and P. J. Sullivan, J. Fluid Mech. 604, 447 (2008).

${ }^{53}$ H. E. Jørgensen, T. Mikkelsen, and H. L. Pécseli, Boundary Layer Meteorol. 137, 345 (2010).

${ }^{54}$ J. A. Krommes, Phys. Plasmas 15, 030703 (2008).

${ }^{55}$ F. Sattin, M. Agostini, R. Cavazzana, G. Serianni, P. Scarin, and N. Vianello, Phys. Scr. 79, 045006 (2009).

${ }^{56}$ I. Bar-David and A. Nemirovsky, IEEE Trans. Inf. Theory 18, 27 (1972).

${ }^{57}$ H. Biermé and A. Desolneux, J. Appl. Probab. 49, 100 (2012).

${ }^{58}$ G. Casella and R. L. Berger, "Statistical inference," in Duxbury Advanced Series, 2nd ed. (Thomson Learning, 2001).

${ }^{59}$ W. Fundamenski, O. E. Garcia, V. Naulin, R. A. Pitts, A. H. Nielsen, J. Juul Rasmussen, J. Horacek, J. P. Graves, and JET EFDA Contributors, Nucl. Fusion 47, 417 (2007).

${ }^{60}$ P. C. Stangeby, Phys. Plasmas 9, 3489 (2002).

${ }^{61}$ W. Fundamenski, W. Sailer, and JET EFDA Contributors, Plasma Phys. Controlled Fusion 46, 233 (2004).

${ }^{62}$ A. Yu. Pigarov, S. I. Krasheninnikov, T. D. Rognlien, M. J. Schaffer, and W. P. West, Phys. Plasmas 9, 1287 (2002).

${ }^{63}$ D. A. D'Ippolito and J. R. Myra, Phys. Plasmas 13, 062503 (2006).

${ }^{64}$ V. Naulin, J. Nucl. Mater. 363-365, 24 (2007).

${ }^{65}$ M. Agostini, J. L. Terry, P. Scarin, and S. J. Zweben, Nucl. Fusion 51 , 053020 (2011).

${ }^{66}$ A. H. Nielsen, H. L. Pécseli, and J. Juul Rasmussen, Ann. Geophys. 10, 655 (1992)

${ }^{67}$ A. H. Nielsen, H. L. Pécseli, and J. Juul Rasmussen, Phys. Plasmas 3, 1530 (1996). 